\title{
Prevalence of Depression Symptoms Before and During the COVID-19 Pandemic Among Two Asian American Ethnic Groups
}

\author{
Paula Lozano $^{1,4}\left(\right.$ D $\cdot$ Sandra Yu Rueger ${ }^{2} \cdot$ Helen Lam $^{1} \cdot$ Naomi Louie $^{3} \cdot$ Alia Southworth $^{3} \cdot$ Chieko Maene $^{3}$. \\ Yicklun $\mathrm{Mo}^{3} \cdot$ Fornessa Randal ${ }^{3} \cdot$ Karen Kim$^{1}$
}

Accepted: 28 September 2021 / Published online: 13 October 2021

(c) The Author(s), under exclusive licence to Springer Science+Business Media, LLC, part of Springer Nature 2021

\begin{abstract}
Asian Americans have experienced compounding stressors during the pandemic as a result of racial discrimination. We aim of to investigate the prevalence of depression symptoms among Asian Americans before and during the COVID-19 pandemic and examine differences based on socio-demographic factors. Data are from a cross-sectional study $(\mathrm{N}=636)$ among Chinese and South Asian adults in Chicago collected between February and May 2020. One cohort of participants were surveyed from each ethnic group before the pandemic and a second cohort of participants were surveyed during the pandemic. Depression symptoms increased more than two-fold, from 9\% pre-pandemic to 21\% during the COVID-19 pandemic. We found an increase in depression symptoms during the pandemic for South Asians, men and adults older than 30 years. These findings call for public health education that effectively addresses anti-Asian harassment and violence and ensure that culturally competent mental health services are provided to Asian Americans from diverse ethnic backgrounds.
\end{abstract}

Keywords Asian Americans · Mental health · Depression symptoms · COVID-19 - Discrimination

\section{Introduction}

The coronavirus 2019 (COVID-19) has resulted in unprecedented changes in the social and economic environment of individuals in the United States (US) and globally [1,2]. COVID-19 was first reported in Wuhan, China and subsequently spread worldwide [3]. On March 13th, 2020, as a result of a drastic increase in infections and deaths across the US, COVID-19 was declared a national emergency. The policies created to prevent the spread of the virus have disrupted the life of most Americans and introduced new life stressors [4]. For instance, stay-at-home advisories or shelter inplace policies across the US (e.g. Illinois stay-at-home order,

Paula Lozano

lozanomesa@medicine.bsd.uchicago.edu

1 University of Chicago Center for Asian Health Equity, Chicago, IL, USA

2 Department of Psychology, Wheaton College, Wheaton, IL, USA

3 Asian Health Coalition, Chicago, IL, USA

4 Center for Asian Health Equity, UChicago Medicine, 5841 S. Maryland Avenue, Rm. A-406, MC4001, Chicago, IL 60637, USA issued on March 21st, 2020) resulted in a drastic increase in unemployment rates [1]. Widespread job loss and furloughs of uncertain durations, along with other COVID-19 related stressors, including social isolation due to quarantining and social distancing guidelines, lack of clear information, and fear of infection, have further contributed to the rise in psychological distress among the US population [4].

Asian Americans in particular have experienced compounding stressors during the pandemic as a result of stigma and racial discrimination [2, 5-11]. As of February 2021, the STOP AAPI Hate campaign had received 3,795 reports of racial discrimination against Asian Americans (i.e., verbal harassment, physical assault and potential civil rights violations) nationwide [12]. Increasing reports of COVID19-related attacks on Asian Americans in the news and social media have perpetuated stigmatizing stereotypes that portray Asians as "perpetual foreigners," "dirty," and "disease-ridden" [2, 5]. In fact, since the COVID-19 outbreak, Asian Americans have been blamed for the spread of COVID-19, referred by many (i.e., political leaders) as the "Wuhan virus," the "Chinese virus," and "Kung Flu" $[3,9,13]$.

Asian Americans are also experiencing racial discrimination in the workplace due to COVID-19 [14, 15]. A recent 
study suggests that less-educated Asian Americans (men and women) were substantially more likely to lose employment than equally educated whites during the COVID-19 lockdown, and less likely to regain employment during the reopening months [15]. Perceived racial discrimination and other COVID-19 related stressors (e.g., losing a job, losing someone close to you from COVID-19, and having financial problems) are associated with increased burden of mental health illness, particularly among members of disadvantaged groups [1-3].

This increase in racial discrimination against Asian Americans related to COVID-19 [2, 5-11] can have serious long-term effects on mental health. In fact, a recent study found a $18.7 \%$ increase in the prevalence of depression symptoms during COVID-19 compared with before COVID-19 among Asian Americas [1]. However, this study used aggregated data that compromise Asians from diverse ethnic backgrounds. Asian Americans are a heterogeneous population that comprise nearly 50 countries and languages. There is a diversity of ethnicities, culture, religion, immigration histories and levels of acculturation among Asian ethnic subgroups $[16,17]$. Therefore, there is a need for data disaggregation to better understand the mental health needs of Asian Americans from different ethnic subgroups.

Related to COVID-19, there has been significant backlash against China (since the pandemic originated in China) that may influence discrimination experiences of those of Chinese heritage compared to other Asian countries of origin. Not surprisingly, studies in China [18] and the US [5] find that direct exposure to COVID-19 (e.g. personal exposure, media exposure, and impact on livelihood) is a risk factor for mental health problems among Chinese and Chinese Americas. However, less is known about the influence of COVID19 exposure on other Asian ethnic subgroups in the US.

Thus, the goal of the current study is to investigate the prevalence of depression symptoms among two ethnic subgroups of Asian Americans to examine differences between two distinct Asian cultural groups. It is possible that Asian Americans from other countries, who are less likely to have social and emotional connections to China, would be less influenced by COVID-19. In addition, it is possible that Asian Americans who have a distinctly different physical appearance compared to those with Chinese ethnic roots, may not be experiencing the same level of discrimination as those from China. Thus, the primary goal of the current study was to compare depression levels in Chinese with South Asians (predominantly Indian and Pakistani) in Chicago, to investigate depression levels experienced by those in these distinct communities both before and during the COVID-19 pandemic. Moreover, a recent national study found differences in depression symptoms before and during COVID-19 by sex, age education marital status and income for the US population [1].
Therefore, a secondary goal is to investigate differences in depression for the Asian American population based on sociodemographic factors that have been found to be associated with higher rates of depression $[19,20]$.

\section{Methods}

\section{Procedures}

The current study involves a secondary analysis of The Chicago Asian Health Survey, a cross-sectional survey conducted by the Asian Health Coalition (AHC). The AHC is a non-profit, grassroots organization focused on improving the health and well-being of Asian Americans through advocacy, technical assistance, education, and community-based research. The primary goal of this survey was to collect self-reported, de-identified disaggregated health information among eight targeted Asian American (i.e. Cambodian, Chinese, Filipino, Korean, Laotian, Mongolian, South Asian, or Vietnamese) communities in Chicago. Of the eight communities in the study, only the Chinese and South Asian communities, who were surveyed over a period of four months (form February 2020 to May 2020) had data both before and during the COVID-19 outbreak. Thus, in this study we use these extant data to examine differences in depression symptoms between Chinese and South Asians.

The data used in the current study were collected using convenience sampling and active partnership between the AHC with four community-based organizations (CBOs), two each within the Chinese and South Asian communities. The CBOs disseminated information about the survey and recruited participants to increase engagement with the research process. Each $\mathrm{CBO}$ was encouraged to recruit participants with strategies they believed would effectively reach their community (e.g., recruitment of participants through community events, word of mouth, social media posts, sharing information with staff and local partners, and calling past clients). While most participants completed the online survey through tablets or computers, participants also had the option of completing a paper version of the survey or a telephone-based survey. The AHC offered to translate the survey to different Asian languages. Only one request was made to translate the survey to Simplified Chinese. After the Illinois stay-at-home order was issued on March 21st, 2020, partner organizations halted in-person events and dissemination efforts, transitioning to virtual recruitment and online survey collection. To ensure unduplicated responses of survey data, our partner CBO cross-referenced emails and names of participants. 


\section{Participants}

Adults (18 years or older) who reside in the Chicagoland area and who self-identify as South Asian (e.g., from India, Pakistan, Bangladesh, Sri Lanka, Afghanistan and Nepal) or Chinese were eligible to participate in the survey. From an initial sample size of 764 participants, we excluded 11 participants who had missing data for sex, 8 participants who had missing data for education, 4 participants who had missing data for acculturation and 105 participants who had answered "I do not know" to at least one of the PHQ-8 items and were coded as missing. The final sample size for this study was 636 ( $n=288$ before COVID-19; $n=348$ during COVID-19) participants. The University of Chicago Institutional Review Board granted approval for this study.

\section{Measures}

\section{Depression Symptoms}

Depression symptoms were assessed using the patient health questionnaire eight-item depression scale (PHQ-8) [21-23]. The PHQ-8 is a self-reported questionnaire that consists of eight of nine items included in the diagnostic criteria for major depressive disorder in the diagnostic and statistical manual of mental disorders, fourth edition (DSM-IV) [21, 22]. A standard cutoff point of 10 was used to assess depression symptoms $[1,22,24,25]$. A previous study suggest that the PHQ- 8 score of $\geq 10$ has a sensitivity of $88 \%$ and specificity of $88 \%$ for major depression [22].

\section{Sociodemographic Variables}

Sociodemographic factors were assessed with singleitem questions in the survey including: Asian ethnic subgroup (Chinese, South Asian), sex (men/women), age (18-29 years, 30 years and more), education (less than high school, high school, some college, college or more), employment (employed, not employed, student), time in the US (Foreign born-0-9 year in the US, Foreign born-10-19 year, Foreign born -20 or more and Us born).

\section{Acculturation}

Acculturation was assessed with a modified version of the Suinn-Lew Asian Self-Identity Acculturation-Item Total Correlation (SLASIA-ITC) [26]. The SLASIA-ITC consists of five items: language spoken, language preferred, language read, childhood friends, and self-assessed level of acculturation (Cronbach's $\alpha=0.88$ ) [26]. This study, which included four (i.e., language spoken, language preferred, language read, and self-assessed level of acculturation) out of the five items in this scale, demonstrated high internal consistency (Cronbach's $\alpha=0.92$ ).

\section{Statistical Analysis}

To test the influence of the COVID-19 pandemic on depression symptoms, we created a time dummy variable distinguishing the period before and after March $13^{\text {th }}$ which represents the date when the US declared the COVID-19 pandemic a national emergency. First we estimated descriptive statistics by Asian ethnic subgroup (i.e., Chinese and South Asians) before and during the COVID-19 outbreak. In addition, the prevalence of depression symptoms by sociodemographic characteristics were evaluated for participants who responded to the survey before and during the COVID-19 pandemic. To assess statistical significance of these comparisons at a p-value of 0.05 , we conducted chi-square tests for categorical variables and ANOVA for continuous variables.

We further conducted unadjusted and adjusted (i.e., sex, age, Asian ethnic subgroup education, employment, time in the US and acculturation, time dummy variable for COVID19) logistic regression models to examine the association between exposure to COVID-19 (i.e., COVID-19 time period dummy variable) and depression symptoms. Then, we tested the moderating effect of the sociodemographic factors on the association between the Covid-19 exposure and depression. The COVID-19 time dummy variable $\mathrm{X}$ sociodemographic interaction terms were tested in separate models. We adjusted for multiple testing using the Benjamini-Hochberg correction method [27] with the false discovery rate at $5 \%$ across the interaction models. If the interaction was statistically significant ( $\mathrm{p}$ value $<0.05$ ), we plotted predicted marginal probabilities of depression symptoms by sociodemographic characteristics, before and during the COVID-19 pandemic. All analyses were conducted in Stata 15.

\section{Result Section}

Descriptive statistics by Asian ethnic subgroup (i.e., Chinese and South Asians) before and during the COVID-19 outbreak are shown in Table 1. Results showed there was statistically significant variation in the distribution of time in the US for Chinese participants, such that there were more US born (27\%) participants before the COVID-19 outbreak compared to during the pandemic (14\%). Among South Asian participants, there was statistically significant variation in the distribution of education, such that $53 \%$ of participants had a college education or more before COVID-19 compared to $32 \%$ after the COVID-19 outbreak. There were no other statistically significant variations in the sample. 
Table 1 Study characteristics by Asian American ethnic groups before and during COVID-19, Chicago Community Health Survey 2020 $(\mathrm{N}=637)$

\begin{tabular}{|c|c|c|c|c|c|c|}
\hline \multirow[t]{3}{*}{ Characteristics } & \multicolumn{2}{|l|}{ Chinese } & \multirow[t]{3}{*}{$\mathrm{p}$ value $^{\mathrm{a}}$} & \multicolumn{2}{|l|}{ South Asian } & \multirow[t]{3}{*}{$p$ value $^{a}$} \\
\hline & Before $(n=150)$ & During $(\mathrm{n}=237)$ & & Before $(n=138)$ & During $(\mathrm{n}=111)$ & \\
\hline & $\mathrm{n}(\%)$ & $\mathrm{n}(\%)$ & & $\mathrm{n}(\%)$ & $\mathrm{n}(\%)$ & \\
\hline Sex, $\%$ & & & 0.287 & & & 0.308 \\
\hline Male & $68(45)$ & $95(40)$ & & $62(45)$ & $55(50)$ & \\
\hline Female & $82(55)$ & $142(60)$ & & $76(55)$ & $56(50)$ & \\
\hline Age group, $\%$ & & & 0.093 & & & 0.929 \\
\hline $18-29$ years & $49(33)$ & $60(25)$ & & $58(42)$ & $44(40)$ & \\
\hline 30 years or more & $101(67)$ & $177(75)$ & & $80(58)$ & $67(60)$ & \\
\hline Education, $\%$ & & & 0.068 & & & 0.013 \\
\hline Less than high school & $53(35)$ & $58(24)$ & & $12(9)$ & $13(12)$ & \\
\hline High school & $22(15)$ & $53(22)$ & & $23(17)$ & $24(22)$ & \\
\hline Some college & $22(15)$ & $32(14)$ & & $30(22)$ & $38(34)$ & \\
\hline College or more & $53(35)$ & $94(40)$ & & $73(53)$ & $36(32)$ & \\
\hline Employment, (\%) & & & 0.093 & & & 0.783 \\
\hline Employed & $72(48)$ & 115 (49) & & $83(60)$ & $62(56)$ & \\
\hline Not employed & $52(35)$ & $98(41)$ & & $30(22)$ & $26(23)$ & \\
\hline Student & $26(17)$ & $24(10)$ & & $25(18)$ & $23(21)$ & \\
\hline Time in the US, (\%) & & & $<0.001$ & & & 0.574 \\
\hline Foreign born- $0-9$ year in the US & $24(16)$ & $91(38)$ & & $48(35)$ & $40(36)$ & \\
\hline Foreign born-10-19 year & $24(16)$ & $39(16)$ & & $42(30)$ & $28(25)$ & \\
\hline Foreign born- 20 or more & $62(41)$ & $75(32)$ & & $29(21)$ & $21(19)$ & \\
\hline Us born & $40(27)$ & $32(14)$ & & $19(14)$ & $22(20)$ & \\
\hline \multirow[t]{2}{*}{ Acculturation, mean (SD) } & & & 0.619 & & & 0.307 \\
\hline & $2.37(1.26)$ & $2.31(1.09)$ & & $3.18(0.94)$ & $3.30(0.94)$ & \\
\hline
\end{tabular}

Bold values indicate $\mathrm{p} \leq 0.05$

Participants surveyed before the COVID-19 pandemic differ from those surveyed during the COVID-19 pandemic

${ }^{\text {a }}$ wwo-tailed $\chi 2$ analysis conducted for significance testing within each Asian ethnic subgroup

We explored the prevalence of depression symptoms by sociodemographic indicators before and during COVID-19 (Table 2). Results suggest that there was over a two-fold increase in depression symptoms during the pandemic compared to before the pandemic (During COVID-19: 21\% vs. Before COVID-19: 9\%). We found that while there were no statistically significant differences in depression symptoms among Chinese and South Asians before COVID-19, the prevalence of depression symptoms was significantly higher for South Asian compared to Chinese during the COVID-19 pandemic (13\% Chinese vs. 38\% South Asians). Likewise, although there was no statistically significant difference in the prevalence of depression symptoms among women and men before COVID-19, men reported more depression symptoms during COVID-19 compared to women (17\% women vs. $26 \%$ men). There also were statistically significant differences, before and during COVID-19 by age, such that younger participants (18-29 years) had a higher prevalence of depression symptoms before COVID-19 compared to participants who were 30 years or older, but there were no statistically significant differences among these two groups during the COVID-19 outbreak. Results suggest that students had a higher prevalence of depression both before and during the pandemic, compared to employed and unemployed participants. Finally, results indicate that although there were no statistically significant differences in depression by acculturation before COVID-19, more acculturated participants had a higher prevalence of depression symptoms during the COVID-19 outbreak.

As expected, results from the unadjusted logistic regression models were similar to our descriptive analysis (Table 2): Asian Americans were more likely to report depression symptoms during COVID-19 compared to before the pandemic (OR 2.74, 95\% CI 1.69-4.46). Importantly, this relationship remained significant after adjusting for sociodemographic indicators (AOR 3.85, 95\% CI 2.28-6.49). The test of moderation of the relationship between exposure to COVID-19 and depression symptom by sociodemographic indicators demonstrated significant effects (Table 3). Specifically, we found a statistically significant interaction 
Table 2 Prevalence of depression symptoms by sociodemographic characteristics before and during Covid-19, Chicago Community Health Survey $2020(\mathrm{~N}=637)$

\begin{tabular}{|c|c|c|c|c|c|c|c|}
\hline \multirow[t]{2}{*}{ Characteristics } & \multicolumn{3}{|c|}{ Before Covid-19 n (\%) } & \multirow[t]{2}{*}{$\mathrm{p}$ value ${ }^{\mathrm{b}}$} & \multicolumn{2}{|c|}{ During Covid-19 n (\%) } & \multirow[t]{2}{*}{$\mathrm{p}$ value ${ }^{\mathrm{b}}$} \\
\hline & \multicolumn{2}{|c|}{ Total $(\mathrm{n}=288)$} & Depression symptoms $^{\mathrm{a}}(\mathrm{n}=25)$ & & Total $(n=348)$ & $\begin{array}{l}\text { Depression } \\
\text { symptoms }^{\mathrm{a}} \\
(\mathrm{n}=72)\end{array}$ & \\
\hline \multicolumn{8}{|l|}{ Asian ethnic subgroup, $\%$} \\
\hline Chinese & $150(52)$ & $11(7)$ & & \multirow[t]{2}{*}{0.397} & $237(68)$ & $30(13)$ & \multirow[t]{2}{*}{$<0.001$} \\
\hline South Asian & $138(48)$ & $14(10)$ & & & $111(32)$ & $42(38)$ & \\
\hline \multicolumn{8}{|l|}{ Sex, $\%$} \\
\hline Male & $130(45)$ & $8(6)$ & & \multirow[t]{2}{*}{0.167} & $150(43)$ & $39(26)$ & \multirow[t]{2}{*}{$\mathbf{0 . 0 3 3}$} \\
\hline Female & $158(55)$ & $17(11)$ & & & $198(57)$ & $33(17)$ & \\
\hline \multicolumn{8}{|l|}{ Age group, \% } \\
\hline $18-29$ years & $107(37)$ & $17(16)$ & & \multirow[t]{2}{*}{0.001} & $104(30)$ & $28(27)$ & \multirow[t]{2}{*}{0.061} \\
\hline 30 years or more & $181(63)$ & $8(4)$ & & & $244(70)$ & $44(18)$ & \\
\hline \multicolumn{8}{|l|}{ Education, $\%$} \\
\hline Less than high school & $65(23)$ & $5(8)$ & & \multirow[t]{4}{*}{0.923} & $71(20)$ & $17(24)$ & \multirow[t]{4}{*}{0.169} \\
\hline High school & $45(16)$ & $3(7)$ & & & $77(22)$ & $12(16)$ & \\
\hline Some college & $52(18)$ & $5(10)$ & & & $70(20)$ & $20(29)$ & \\
\hline College or more & $126(44)$ & $12(10)$ & & & $130(37)$ & $23(18)$ & \\
\hline \multicolumn{8}{|l|}{ Employment } \\
\hline Employed & $155(54)$ & $9(6)$ & & \multirow[t]{3}{*}{0.034} & $177(51)$ & $38(21)$ & \multirow[t]{3}{*}{0.005} \\
\hline Not employed & $82(28)$ & $7(9)$ & & & $124(36)$ & $17(14)$ & \\
\hline Student & $51(18)$ & $9(18)$ & & & $47(14)$ & $17(36)$ & \\
\hline \multicolumn{8}{|l|}{ Time in the US } \\
\hline Foreign born-0-9 year in the US & $74(25)$ & $5(7)$ & & \multirow[t]{4}{*}{0.029} & $128(37)$ & $18(14)$ & \multirow[t]{4}{*}{0.088} \\
\hline Foreign born-10-19 year in the US & $66(23)$ & $7(11)$ & & & $67(19)$ & $15(22)$ & \\
\hline Foreign born- 20 or more in the US & $92(32)$ & $3(3)$ & & & $97(28)$ & $25(26)$ & \\
\hline Us born & $60(21)$ & $10(17)$ & & & $53(15)$ & $14(26)$ & \\
\hline \multicolumn{8}{|l|}{ Acculturation $^{c}$} \\
\hline No & $144(50)$ & $6(6)$ & & \multirow[t]{2}{*}{0.060} & $198(57)$ & $29(15)$ & \multirow[t]{2}{*}{0.001} \\
\hline Yes & $144(50)$ & $17(12)$ & & & $150(43)$ & $43(29)$ & \\
\hline
\end{tabular}

Participants surveyed before the COVID-19 pandemic differ from those surveyed during the COVID-19 pandemic

Bold font in stats indicate $\mathrm{p}$ values $\leq 0.05$

${ }^{a}$ Prevalence of depression symptoms by sociodemographic indicators

${ }^{\mathrm{b}}$ Two-tailed $\chi 2$ analysis conducted for significance testing

${ }^{\mathrm{c}}$ Acculturation was assessed as the median split of the continuous measure used in this study

between the time period dummy variable and Asian American ethnic subgroups, such that the predictive probability of depression symptoms was comparable among Chinese and South Asians before COVID-19, but was higher for South Asian during the pandemic (Fig. 1a). We also found a statistically significant interaction between the time period dummy variable and sex, indicating that the predictive probability of depression symptoms was higher for women compared to men before COVID-19 but was higher for men during the pandemic (Fig. 1b). Finally, we found a statistically significant interaction between the time period dummy variable and age groups), such that while younger participants (i.e., 18-29 years) were more likely to report depression symptoms compared to their older counterpart (i.e.; 30 years and older) before COVID-19, the predictive probability of depression symptoms was comparable among these two groups during the pandemic (Fig. 1c). No other analyses were statistically significant.

\section{Discussion}

Consistent with a previous national study, we found that depression symptoms increased more than two-fold, from 9\% pre-pandemic to $21 \%$ during the COVID-19 pandemic among Asian Americans (i.e., Chinese and South Asians) in 
Table $3 \mathrm{P}$ values for joint test of multiplicative interactions between COVID-19 time variable and sociodemographic for depression symptoms, Chicago Community Health Survey $2020(\mathrm{~N}=637)$

\begin{tabular}{ll}
\hline Interaction $^{\mathrm{a}}$ & $\begin{array}{l}\text { Depression } \\
\text { symptoms }\end{array}$ \\
\hline COVID-19 time variable $\times$ Asian ethnic group & $0.0375^{\mathrm{b}}$ \\
COVID-19 time variable $\times$ Sex & $0.025^{\mathrm{b}}$ \\
COVID-19 time variable $\times$ Age group & $0.0125^{\mathrm{b}}$ \\
COVID-19 time variable $\times$ Education & 0.7640 \\
COVID-19 time variable $\times$ Employment & 0.2933 \\
COVID-19 time variable $\times$ Time in the US & 0.05 \\
COVID-19 time variable $\times$ Acculturation & 0.8878 \\
\hline
\end{tabular}

Participants surveyed before the COVID-19 pandemic differ from those surveyed during the COVID-19 pandemic

${ }^{a}$ Each interaction was estimated from a separate logistic regression model with all main effects and a single interaction term between Covid-19 time variable and sociodemographic variable. Models were adjusted for sociodemographic indicators

${ }^{\mathrm{b}}$ Indicates significance after controlling for false discovery rate at $5 \%$

Chicago [1]. However, disaggregated data by Asian American ethnic subgroups suggest that while the prevalence of depression symptoms was similar for Chinese and South Asians before the pandemic, there was a dramatic increase in the prevalence of depression symptoms for South Asians during the pandemic. Results are consistent with a recent Canadian study that found that $22 \%$ of South Asian participants consider that COVID-19 had impacted their mental health "a great deal" compared to $11 \%$ of East Asians (e.g. Chinese) [28]. Differences in depression symptoms during the COVID-19 pandemic among South Asians and Chinese may be attributed to a combination of factors. First, it is possible that Chinese participants who have communication with family and friends in China were made aware of the unprecedented changes in the social environment that resulted due to COVID-19 earlier in the pandemic and were therefore more prepared to cope with COVID-19 related stressors compared to South Asians. Second, cultural norms around reporting mental health issues (i.e., Chinese may be less likely to express distress than South Asian) may also differ by Asian American ethnic subgroups [28]. Future studies need to examine differences in mental health related stigma among Asian Americans from diverse ethnic background. Third, it is possible that Asian ethnic enclaves such as Chicago's Chinatown, that develop quickly due to chain migration, may act as a protective environment for immigrant communities $[17,29]$. In fact, a recent study among Asian Americans in the US indicated that participants who did not experience discrimination during the COVID19 outbreak attributed this to the fact that the community where they lived in was predominately Asian [11]. Thus, it is possible that there were fewer non-Asian individuals to a

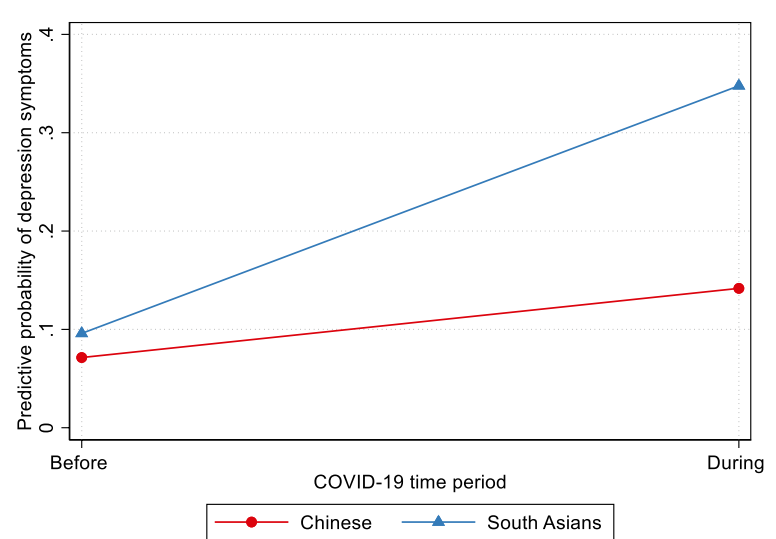

b

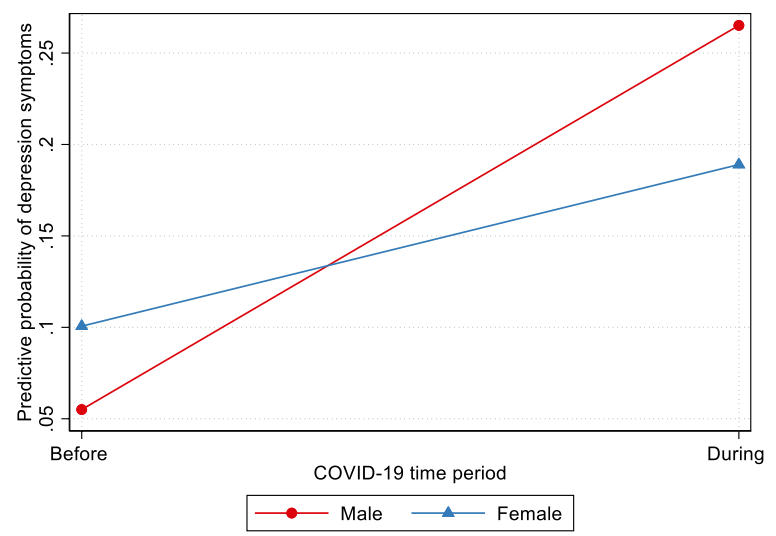

C

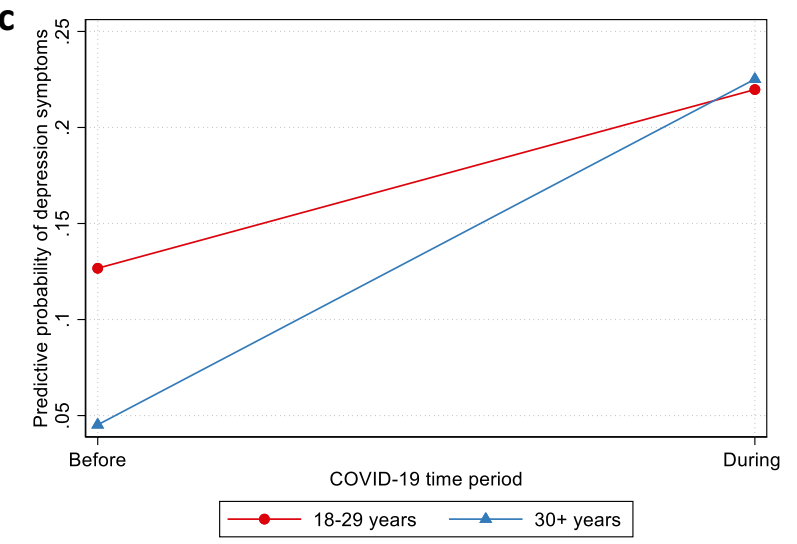

Fig. 1 Predicted probability of depression symptoms by (a) Asian Americans ethic group (b) sex and (c) age group. Chicago Community Health Survey 2020. ( $=637) *$ Participants surveyed before the COVID-19 pandemic differ from those surveyed during the COVID19 pandemic

commit discriminatory acts or that people who might otherwise engage in discriminatory acts were less likely to when Asian Americans are not minority members of their communities [11]. The South Asians in this sample (i.e., India and Pakistan, Bangladesh, Sri Lanka, Afghanistan and Nepal) may have less established ethnic neighborhood communities 
in Chicagoland. Thus, compared to the South Asian participants, the Chinese participants may have benefitted from a more culturally cohesive community and social support embedded within Chicago's Chinatown neighborhoods.

This study also found that depression symptoms were higher for Asian American women compared to men before COVID-19 but higher for men during the pandemic. Recent findings suggest that women (particularly mothers) were more likely to exit the labor force and become unemployed $[14,30,31]$ during the pandemic. Thus, it is possible that Asian American women staying at home were less exposed to COVID-19 related racial attacks, and prejudice compared to Asian American men. Furthermore, it is possible that depression symptoms were higher for Asian American men compared to Asian American women during the pandemic, due to COVID-19 related stressors that result from unemployment as well as discrimination in the workplace [14, 15]. In fact, a study suggest that unemployment was higher for Asian American (particularly men) compared to people from other racial/ethnic subgroups during the COVID-19 lockdown [15].

Finally, we found that although younger participants (i.e., 18-29 years) were more likely to report depression symptoms compared to their older counterpart (i.e.; 30 years and older) before COVID-19, the predictive probability of depression symptoms was comparable among these two groups during the pandemic. Studies suggest that emerging adults (i.e. individuals within the age of 18-29) are more likely to report depressive and behavioral disorders compared to other age groups [32-34]. Therefore, it is not surprising that before the pandemic this age groups (i.e., 18-29 yeas) reported more depressive symptoms compared to participants 30 years and older. Similar to the gender difference finding, it is possible that the detected increase in depression symptoms among participants 30 years and older during the pandemic may have arisen due to increased exposure to work place discrimination during COVID-19. In fact, our study suggests that $74 \%$ of employed participants were between the age of 30 and 65 . Future studies, need to examine the relationship between COVID-19 related discrimination in the workplace and mental health outcomes.

\section{Strengths and Limitations}

Strengths of this study include a diverse sample, including Asian Americans with low socioeconomic status, limited English proficiency and low heath literacy that are generally excluded from nationally representative health surveys (e.g., NHANES) [35, 36]. In fact, this survey includes a higher percentage of participants with less than a high school education compared to the nationally representative sample reports by the US Census Bureau (12\% vs. $21 \%$ ) [37]. Another strength of the study is the use of disaggregated ethnic data, which allowed for comparisons between two Asian American subgroups. Future studies investigating a comparison of Asian American subgroups to other ethnic/racial minorities would add to our understanding of differences in experiences of and response to discrimination across a broader range of racial/ ethnic subgroups. Limitations of this study included the use of cross-sectional data with two distinct cohorts of participants (from each ethnic subgroup) surveyed before COVID-19 and during the pandemic. Therefore, results for this study should be interpreted at the target population level and not at the individual level. The use of a convenience sample, is another limitation that affects the generalizability of our results. However, studies suggest that when recruitment is done in partnership with local ethnic venues, convenience sampling can yield a representative sample [35, 38]. For instance, a study among Korean and Chinese Americans used two sampling methods (random sampling vs. convenience sampling) to recruit participants and found no large, statistically significant differences in participants' demographic characteristics [35, 38]. Finally, although, COVID-19 was declared a national emergency on March 13th, (cutoff for this study) it is possible that Asian Americans may have started to experience COVID19 related stressors during the month of February, such as stress related to global air travel restrictions, lack of clear information, and fear of infection. This misclassification may have introduced bias towards the null, especially with the Chinese subsample.

\section{Conclusions}

To date, there is limited data examining the impact of COVID-19 related stressors (e.g., unemployment, discrimination) among Asian Americans of diverse ethnic backgrounds. Asian Americans have experienced compounding stressors during the pandemic as a result of anti-Asian stigma and discrimination [2,5-11], which may have serious long-term effects on their mental health. Results from this study suggest that there was a significant increase in the prevalence of depressive symptoms among South Asians during the COVID-19 pandemic. This is particularly concerning considering that Asian Americans are less likely to seek mental health care or treatment compared to other racial/ethnic subgroups [39]. Further, results showed gender and age-related differences that could be attributed to increased discrimination in the workplace. These findings call for public health education that effectively addresses anti-Asian harassment and violence and ensure that culturally competent mental health services (e.g., designed to 
address low levels of mental health literacy) are provided to Asian Americans from diverse ethnic backgrounds.

Author Contributions All authors have approved the manuscript and agreed with its submission to the journal.

Funding This study was funded by Blue Cross and Blue Shield of Illinois (BCBSIL).

\section{Declarations}

Conflict of interest The authors declare that they have no conflict of interest.

Ethical Approval The University of Chicago Institutional Review Board granted approval for this study.

\section{References}

1. Ettman CK, Abdalla SM, Cohen GH, Sampson L, Vivier PM, Galea S. Prevalence of depression symptoms in US adults before and during the COVID-19 pandemic. JAMA Netw Open. 2020;3(9):e2019686.

2. Misra S, Le PD, Goldmann E, Yang LH. Psychological impact of anti-Asian stigma due to the COVID-19 pandemic: a call for research, practice, and policy responses. Psychol Trauma. 2020;12:461

3. Demirtaş-Madran HA. Exploring the motivation behind discrimination and stigmatization related to COVID-19: A social psychological discussion based on the main theoretical explanations. Front Psychol. 2020. https://doi.org/10.3389/fpsyg.2020.569528.

4. Holingue C, Badillo-Goicoechea E, Riehm KE, Veldhuis CB, Thrul J, Johnson RM, et al. Mental distress during the COVID-19 pandemic among US adults without a pre-existing mental health condition: findings from American trend panel survey. Prev Med. 2020;139:106231.

5. Cheah CS, Wang C, Ren H, Zong X, Cho HS, Xue X. COVID-19 racism and mental health in Chinese American families. Pediatrics. 2020. https://doi.org/10.1542/peds.2020-021816.

6. Wu C, Qian Y, Wilkes R. Anti-Asian discrimination and the Asian-white mental health gap during COVID-19. Ethn Racial Stud. 2020. https://doi.org/10.2139/ssrn.3626460.

7. Yang C-C, Tsai J-Y, Pan S. Discrimination and well-being among Asians/Asian Americans during COVID-19: The role of social media. Cyberpsychol Behav Soc Netw. 2020;23(12):865-70.

8. Yu N, Pan S, Yang C, Tsai J. Exploring the role of media sources on COVID-19-related discrimination experiences and concerns among asian people in the United States: cross-sectional survey study. J Med Intern Res. 2020;22(11):e21684.

9. Wang D, Gee GC, Bahiru E, Yang EH, Hsu JJ. Asian-Americans and Pacific Islanders in COVID-19: emerging disparities amid discrimination. J Gen Intern Med. 2020;35(12):3685-8.

10. Litam SDA. "Take Your Kung-Flu Back to Wuhan": counseling Asians, Asian Americans, and Pacific Islanders with race-based trauma related to COVID-19. Prof Couns. 2020;10(2):144-56.

11. Lee S, Waters SF. Asians and Asian Americans' experiences of racial discrimination during the COVID-19 pandemic: Impacts on health outcomes and the buffering role of social support. Stigma Health. 2020;6:70
12. STOP AAPI HATE. STOP AAPI HATE NATIONAL REPORT 3.19.20 - 8.5.20.

13. Le TK, Cha L, Han H-R, Tseng W. Anti-Asian xenophobia and Asian American COVID-19 disparities. Am Public Health Assoc. 2020;110:1371.

14. Kantamneni N. The impact of the COVID-19 pandemic on marginalized populations in the United States: a research agenda. Amsterdam: Elsevier; 2020.

15. Kim AT, Kim C, Tuttle SE, Zhang Y. COVID-19 and the decline in Asian American employment. Res Soc Stratif Mobil. 2021;71:100563.

16. Chen JA, Zhang E, Liu CH. Potential impact of COVID-19related racial discrimination on the health of Asian Americans. Am J Public Health. 2020;110(11):1624-7.

17. Leong F, Park YS, Kalibatseva Z. Disentangling immigrant status in mental health: Psychological protective and risk factors among Latino and Asian American immigrants. Am J Orthopsychiatry. 2013;83(2pt3):361-71.

18. Guo J, Feng XL, Wang XH, van IJzendoorn MH. Coping with COVID-19: Exposure to COVID-19 and negative impact on livelihood predict elevated mental health problems in Chinese adults. Int J Environ Res Public Health. 2020;17(11):3857.

19. Lee J, Lei A, Sue S. The current state of mental health research on Asian Americans. J Hum Behav Soc Environ. 2000;3(3-4):159-78.

20. Kalibatseva Z, Leong FT. Depression among Asian Americans: review and recommendations. Depress Res Treat. 2011. https:// doi.org/10.1155/2011/320902.

21. Shin C, Lee S-H, Han K-M, Yoon H-K, Han C. Comparison of the usefulness of the PHQ-8 and PHQ-9 for screening for major depressive disorder: analysis of psychiatric outpatient data. Psychiatry Investig. 2019;16(4):300.

22. Kroenke K, Strine TW, Spitzer RL, Williams JB, Berry JT, Mokdad AH. The PHQ-8 as a measure of current depression in the general population. J Affect Disord. 2009;114(1-3):163-73.

23. Razykov I, Ziegelstein RC, Whooley MA, Thombs BD. The PHQ-9 versus the PHQ-8-is item 9 useful for assessing suicide risk in coronary artery disease patients? Data from the heart and soul study. J Psychosom Res. 2012;73(3):163-8.

24. Kroenke K, Spitzer RL, Williams JB. The PHQ-9: validity of a brief depression severity measure. J Gen Intern Med. 2001;16(9):606-13.

25. Corson K, Gerrity MS, Dobscha SK. Screening for depression and suicidality in a VA primary care setting: 2 items are better than 1 item. Am J Manage Care. 2004;10(11 Pt 2):839-45.

26. Leong F, Chou E. Developing brief versions of the SuinnLew Asian selfidentity acculturation (SLASIA) scale for counseling research. Asian Am Pac Isl J Health. 1998;6(1):13-24.

27. Benjamini Y, Hochberg Y. Controlling the false discovery rate: a practical and powerful approach to multiple testing. J R Stat Soc. 1995;57(1):289-300.

28. Miconi D, Li ZY, Frounfelker RL, Santavicca T, Cénat JM, Venkatesh V, et al. Ethno-cultural disparities in mental health during the COVID-19 pandemic: a cross-sectional study on the impact of exposure to the virus and COVID-19-related discrimination and stigma on mental health across ethno-cultural groups in Quebec (Canada). BJPsych Open. 2021. https://doi.org/10. 1192/bjo.2020.146.

29. Liu M, Geron K. Changing neighborhood: ethnic enclaves and the struggle for social justice. Soc Justice. 2008;35(2):18-35.

30. Alon TM, Doepke M, Olmstead-Rumsey J, Tertilt M. The impact of COVID-19 on gender equality: National Bureau of Economic Research. 2020. Report No.: 0898-2937.

31. Landivar LC, Ruppanner L, Scarborough WJ, Collins C. Early signs indicate that COVID-19 is exacerbating gender inequality in the labor force. Socius. 2020;6:2378023120947997. 
32. Kuwabara SA, Van Voorhees BW, Gollan JK, Alexander GC. A qualitative exploration of depression in emerging adulthood: disorder, development, and social context. Gen Hosp Psychiatry. 2007;29(4):317-24.

33. Kessler RC, Walters EE. Epidemiology of DSM-III-R major depression and minor depression among adolescents and young adults in the national comorbidity survey. Depress Anxiety. 1998;7(1):3-14.

34. Klerman GL, Weissman MM. Increasing rates of depression. JAMA. 1989;261(15):2229-35.

35. Holland AT, Palaniappan LP. Problems with the collection and interpretation of Asian-American health data: omission, aggregation, and extrapolation. Ann Epidemiol. 2012;22(6):397-405.

36. Islam NS, Khan S, Kwon S, Jang D, Ro M, Trinh-Shevrin C. Methodological issues in the collection, analysis, and reporting of granular data in Asian American populations: historical challenges and potential solutions. J Health Care Poor Underserved. 2010;21(4):1354.

37. Hoeffel E, Rastogi S, Kim MO, Shahid H. The Asian population: 2010. 2010 Census briefs, 2012. Washington DC: US Census Bureau; 2010.

38. Lee S-K, Cheng Y-Y. Reaching Asian Americans: sampling strategies and incentives. J Immigr Minor Health. 2006;8(3):245-50.

39. Substance Abuse Mental Health Services Administration. Racial/ ethnic differences in mental health service use among adults. Rockville: Substance Abuse and Mental Health Services Administration; 2015.

Publisher's Note Springer Nature remains neutral with regard to jurisdictional claims in published maps and institutional affiliations. 